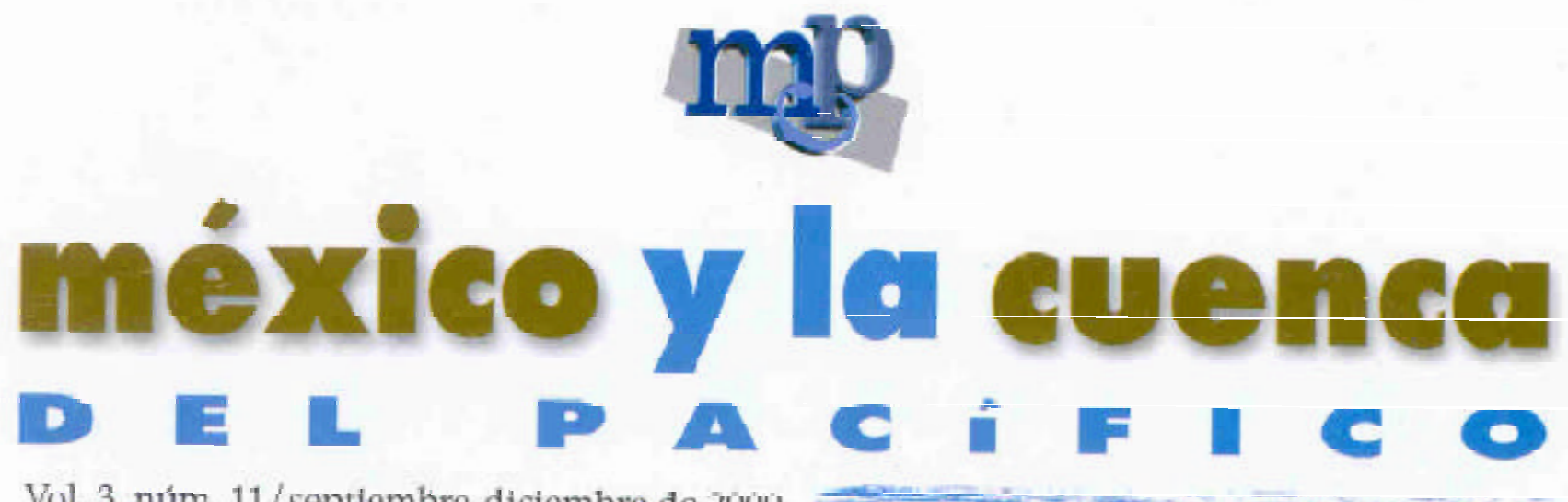

Vol. 3, num. 11/ septiembre-diciembre de 2000
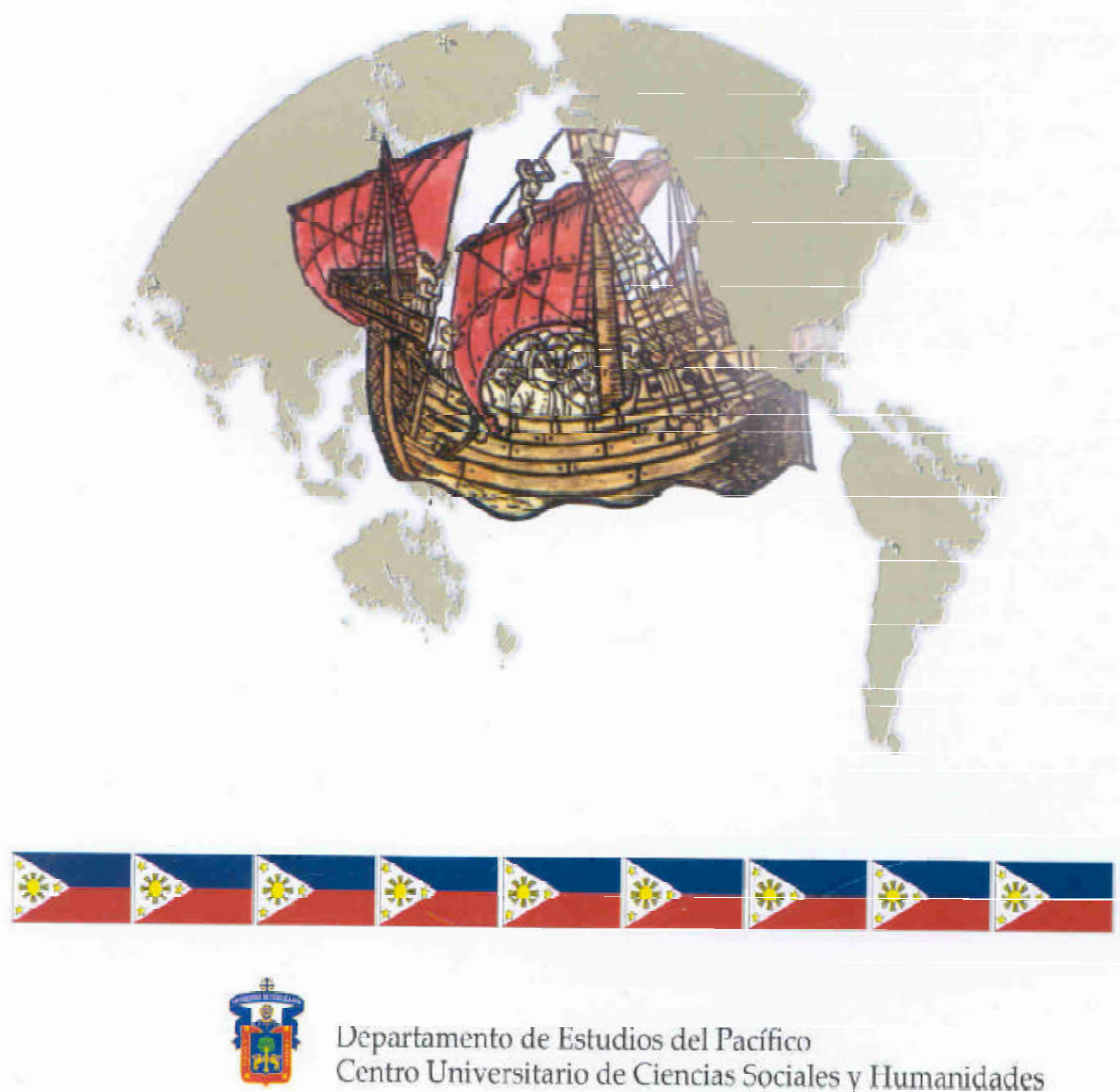

Departamento de Estudios del Pacífico

Centro Universitario de Ciencias Sociales y Humanidades 


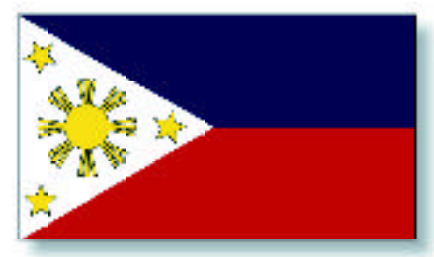

\section{La bandera} de Filipinas

T.a bandera nocional de Filipinas tiene un triángulo blanen at la izquienda. Tiene des franjas: la de a rriba es azul y la de abajo es roja. El triángulo blanco tiene tras estrellas de cinco puntas $y$ un sol con ocho ralyos.

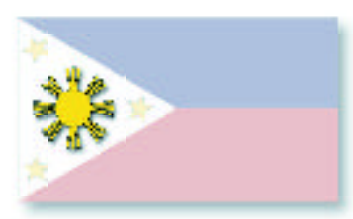

Elsol

Simbolize libertad $y$ sus octus rayls reprutentar las acho primeras provinats tue se rejelaror cor:tra la cononization espanola.

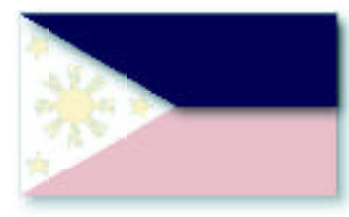

La franja azul

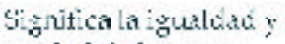
urudad de la gerte.

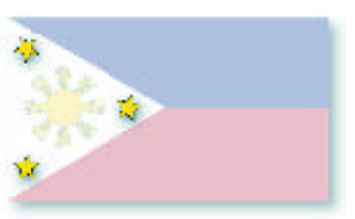

Las estrellas

Representan la división grtogreítice de lilipinusen trex ixertci: I woon, dis Visayas y Mundanzo.

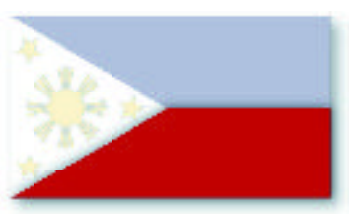

La Lranjạa roja

Simlrolizá la

dispunililidad de lus filipino: de luchar hasta

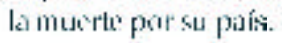


Universidad DE GUADALAJARA

Rector General

Lic. José Trinidad Padilla López

Vicerrector Ejecutivo

Mtro. Ricardo Gutierrez Padilla

Secretario General

Mtro. Carlos Jorge Briseño Torres

Centro Universitario de Ciencias

Sociales y Humanidades

Rector

Dr. Juan Manuel Durán Juárez

Director de la División de

Estudios de Estado y Sociedad

Mtro. Carlos Barba Solano

Jefe del Departamento de Estudios del Pacífico

Mtra. Melba E. Falck Reyes

Septiembre - diciembre, 2000

D.R. (C) 2000, Universidad de Guadalajara

Departamento de Estudios del Pacífico

Av. de los Maestros esq. Av. Alcalde, primer piso,

Ala poniente, edificio ex facultad de Derecho

44260, Guadalajara, Jalisco, México

ISSN: 1665-0174

Impresión

Universidad De GuadaLAJARA

Centro Universitario de Ciencias Social es y Humanidades

Coordinación de Extensión

Guanajuato 1045, Sector Hidalgo

44260, Guadalajara, Jalisco, México

Impreso y hecho en México

Printed and made in Mexico 


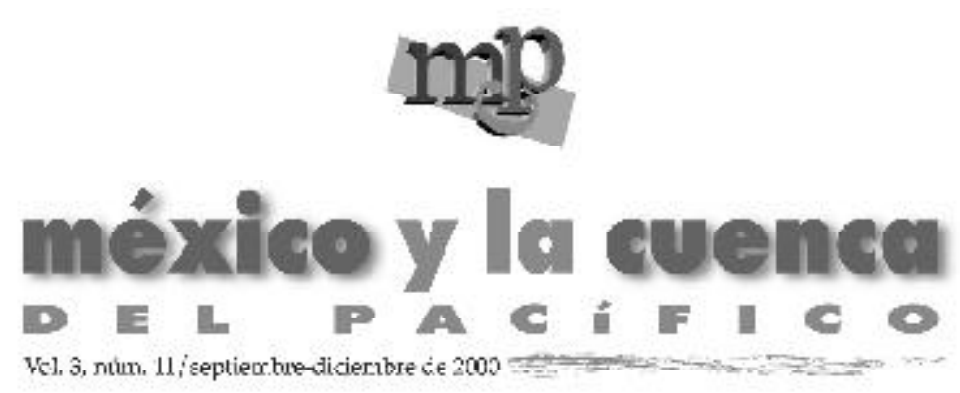

Presentación

Análisis

De la independencia

a la democracia en Filipinas

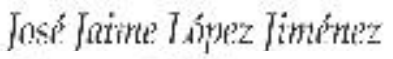

Crisis política en Filipinas

Arturo Santa Cruz

La economia de Filipinas

Melba E. Falck

La política agrícola de Filipinas

Rober to Hernández

Dos crisis financicras y su impacto

sobre el sistema bancario filipino:

$1981-1987$ y 1997-200र1

Geneviève Marchini

Perspectiva histórica del encuentro

de México con Asia-Pacífico:

el caso de Filipinas

Dagoberto Ampuro Tello

El comercio de Filipinas

y su relación con México

Agustina Rodriguez Alegría

Influencia cultural de

la religión católica en Filipinas

Ramón Robledo Padilla

Filipinas y Japón: dos hermanos

que no lo parecen

Silvia Nozelo Urdanivid

ONG y pobreza rural:

lecciones de Filipinas

Susana A. Preciado Jiménez
4

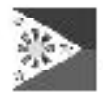

Temas varios del Pacífico

La mujer en la sociedad coreana

58

Maráa Alejardara Isón Garía

El modelo japonés de producción

64

7

y la salud obrera. La polémica actual

Luis Pérez Garcia

La región del Pacífico Mexicano

71

12

en la Cuenca del Pacífico

Meiba E. Falck.

14

21

27

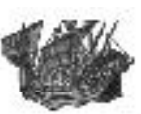

Cooperación internacional

en la Cuenca del Pacífico

La participación de Filipinas en los

75

organismos de cooperación de la

Cuenca del Pacífico

Digoberto Amparo Telio

$3 \overline{5}$

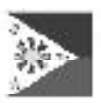

Noticias de la

Cuenca del Pacífico

Beatriz Carrillo

39

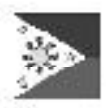

Ecos de Asia en México

Yesmith Ortege

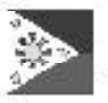

Nuevas adquisiciones

bibliográficas del DEP

Yesmith Ortege

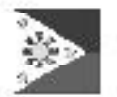

Actividades

87
52 


\section{Presentación}

DOI: $10.32870 /$ mycp.v3i11.106

$\mathrm{E}$ ste número de la revista México y la Cuenca del Pacífico está dedicado a Filipinas, país que desde tiempos de la colonia ha mantenido vínculos estrechos con México. Diez ensayos abordan este país desde diferentes perspectivas: la política, la economía; las relaciones con México; la influencia extranjera sobre la cultura y el comercio filipino; la participación de la sociedad civil filipina en el desarrollo del país.

Los dos artículos iniciales están dedicados al análisis de la esfera política del archipiélago. El primero hace un recuento histórico del p r o c e s o independentista deFilipinas, que después de cuatro siglos de dominio español y cuatro décadas bajo el norteamericano, logra su independencia en 1946. Sin embargo, el fin del coloniaje no significó para esta nación el fin de sus problemas políticos. La i nci piente democracia dio paso a la dictadura de dos décadas de Ferdinand Marcos. A partir de mediados de los ochenta, Filipinas ha tenido tres gobi ernos electos, tratando de consolidar su democracia. El último de éstos, el de Estrada, ha atravesado por una fuerte crisis de credibilidad, tema abordado por el segundo artículo dedicado a los aspectos políticos.

Un segundo bloque de artículos analiza la esfera económica de Filipinas, país que cuenta con 76 millones de habitantes y cuya economía representa una cuarta parte del tamaño de la mexicana. El primero de los ensayos de este grupo analiza la evolución de la economía filipina a partir de la década de los sesenta, destacando que aunque Filipinas era en esa época el país que se perfilaba como el que tomaría la delantera en el sendero del crecimi ento con respecto a sus vecinos en el Pacífico Asiático, paradójicamente, ha constituido una de las excepciones de la historia de éxito de Asia Pacífico.

Una de las características de la economía filipina es todavía su fuerte dependencia del sector agrícola, tanto en términos de producción como de generación de empleo. Es por ello que en otro de los ensayos se analiza con detalle el alcance de la política agrícola en este país y los problemas que ha enfrentado en su aplicación.

Otro sector importante en la economía filipina, el sector financiero, es estudiado en un tercer artículo, que analiza con detalle las dos crisis financieras que ha enfrentado Filipinas, una en los ochenta, que causó una crisis bancaria de carácter sistémico, y la otra, en los noventa, que fue menos aguda y generalizada. Se presenta también un análisis comparativo con las respectivas crisis de México (1982 y 1994). 
Las relaciones de México con Filipinas son abordadas en dos artículos. EI primero presenta un recuento histórico de las relaciones de los dos países desde la época de la colonia, cuando el "tornaviaje" de Oriente al puerto de La Navidad en la Nueva España, realizado por Andrés de Urdaneta, sentó las bases para el intercambio comercial y cultural entre Filipinas y México. Un segundo artículo está dedicado al comercio de Filipinas y su relación con México en la actualidad.

La influencia cultural y comercial que ha ejercido E spaña y J apón sobre el archipiélago filipino, la primera en un largo período que va de 1521 a 1898 y la segunda, menos profunda pero que también inició en el siglo XVI con el intercambio comercial, aunque tuvo un efecto más pronunciado en el corto período de dominación japonesa entre 1942 y 1945, es analizada en el siguiente grupo de artículos. EI primero se centra en el efecto que el dominio de la religión católica tuvo sobre la educación el desarrollo científico en Filipinas, y el segundo hace un recuento histórico de las relaciones entre Filipinas y J apón.

Cierra la sección de análisis un artículo dedicado a la influencia que las Organizaciones no Gubernamentales (ONG) han tenido en los programas de alivio a la pobreza rural, debido a la importancia del sector agrícola en Filipinas y la fuerte desigualdad en la distribución del ingreso en ese sector. Este ensayo hace hincapié no sól o en la importancia de la organización de la sociedad para reclamar los derechos de una vida digna de la población rural ante un estado incapaz de resolver los problemas de pobreza, sino también en la participación activa de la sociedad proponiendo políticas congruentes con las necesidades más apremiantes.

La sección destinada a temas varios del Paćfico incluye en esta ocasión tres ensayos: dos dedicados a dos países del noreste de Asia, Corea del Sur y J apón, y otro dedicado a la región del Pacífico mexicano en la Cuenca del Pacífico. El tema del papel de la mujer en la sociedad coreana es analizado en el primer ensayo de este grupo. La autora presenta un recuento histórico de la posición social de la mujer desde la dinastía Choson hasta nuestros días, destacando la influencia de las ideas confucionistas que mantuvieron a la mujer coreana bajo un estado de discriminación tanto en el hogar como el trabajo. Sin embargo, en la segunda mitad del siglo XX, cuando Corea del Sur alcanzó el desarrollo industrial, el cambio estructural de la economía, ha propiciado una mayor igualdad de género.

El artículo dedicado a J apón en esta sección aborda el tema de la «producción ligera» al estilo japonés y su influencia sobre la salud de los trabajadores. El autor describe con detalle en qué consiste el sistema de «producción flexible»y «justo a tiempo»y ofrece evidencia presentada por especialistas en el tema, sobre los efectos nocivos en la salud de los obreros. Ello en contraposición a otras posturas que privilegian este método de producción frente al taylorismo y fordismo.

Conduye esta sección un ensayo dedicado a analizar la inserción de la región del Pacífico Mexicano en la Cuenca del Pacífico, resaltando las características más importantes de la región mexicana y las oportunidades, aún inexploradas, que se le presentan al otro lado del Pacífico.

En la sección destinada a los procesos de cooperación en la Cuenca del Pacífico, se incluye un artículo que analiza la participación activa de Filipinas en la integración del grupo de las $\mathrm{N}$ aciones del Sudeste Asiático (ASEAN), establecido en 1967, y para el cual Filipinas era el modelo a seguir, ya que en ese entonces contaba con una economía fuerte, una fuerza de trabajo educada y que hablaba inglés, un sector empresarial sólido y una sociedad civil fuerte.

En la sección referente a las noticias en la Cuenca del Paćfico destacan las económicas: en el plano comercial el avance en la concreción de un tratado entre Singapur y J apón y la normalización de las relaciones comerciales entre China y Estados Unidos; Ias secuelas de la crisis de Asia, la quiebra de la Empresa Daewoo de Corea del Sur y el incremento del 
Presentación

comercio intrarregional, excluyendo a J apón y desplazando a Estados Unidos.

En la sección de ecos de Asia en México destaca también el plano comercial, con las reuniones preliminares entre México y Singapur para establecer un tratado de libre comercio y el apoyo de México a la entrada de China a la OMC, que había sido condicionada por nuestro país. A lo anterior hay que agregar que México tendrá la presidencia del Foro de Cooperación Económica de Asia Pacífico (APEC) en 2002, lo cual significa que recibirá a los líderes de las 21 naciones integrantes del organismo asiático.

En la sección de nuevas adquisiciones bibliográficas de la Unidad de Documentación e I nformación (UDI) del Departamento de Estudios del Pacífico, resaltan varios volúmenes sobre la región de Asia Paćfico, sobre los Cuatro Tigres y sobre Indonesia y Malasia. Con el nuevo material que hemos recibido, Ia UDI cuenta con 1412 libros especializados sobre la Cuenca del Pacífico y 6700 ejemplares de revistas especializadas, 5000 de los cuales son extranjeras.

Finalmente, en la sección de actividades académicas del DEP sobresalen en este período la organización de la presentación fotográfica "Kioto: capital ancestral" que se desarrolló en el marco del vigésimo aniversario del hermanamiento de las ciudades de Guadalajara y Kioto. Contamos con la participación de la Fundación J apón en México y de la Secretaría de Cultura del Estado. Cabe destacar el estrechamiento de los vínculos de colaboración con la Fundación J apón, que realizó la donación de un lote de libros a la UDI del DEP. Tị 